biogramma è stato determinato in terreno solido secondo Kirby-Bauer. Sono stati impiegati dischetti di: Amikacina, Gentamicina, Aztreonam, Cefotaxime, Ceftazidime, Cefepime, Imipenem, Meropenem, Piperacillina, Piperacillina-Tazobactam, Ciprofloxacina, Levofloxacina oltre a Cloramfenicolo e Trimetoprim-sulfa per Stenotrophomonas maltophilia. Gli aloni d'inibizione sono stati interpretati secondo le regole NCCLS.

Risultati e conclusioni

Per quanto riguarda $P$ s.aeruginosa $72 \times 2$ pannelli hanno fornito 141 ID corrette con un'accuratezza a livello di specie del $97.9 \%$ e una riproducibilità delle replicle pari al $95.7 \%$. St. maltophilia $26 \times 2$ pannelli hanno fornito 50 ID corrette con un'accuratezza a livello di specie del $96.2 \%$ e una riproducibilità delle repliche pari al $92.3 \%$.

Per $A$. lwoffii e baumanii $14 \times 2$ e $9 \times 2$ pannelli hanno fornito rispettivamente 24 e 17 corrette ID con la presenza di alcuni errori di ID a livello di specie, ma non di genere e riproducibilità delle repliche pari al 71.5 e $88.8 \%$. Da considerare che gli errori più frequentemente riscontrati sono stati errori dovuti alla mancata identificazione del ceppo (7 su 242 pannelli)

Anche per i risultati relativi all'esecuzione dell'antibiogramma Phoenix ha dimostrato estrema accuratezza. Le repliche hanno evidenziano grande riproducibilità dei dati. Le rare discrepanze riscontrate rispetto Kirby-Bauer hanno interessato soltanto intervalli Sensibile-Intermedio o IntermedioResistente Eccezionali sono le discrepanze SensibileResistente.

\section{G060 \\ RIVALUTAZIONE DELLA PREVALENZA DI GARDNERELLA,TRICHOMONAS VAGINALIS E CANDIDA NELL'ESSUDATO VAGINALE CON SONDE MOLECOLARI}

Casari E., Ferrario A., Cristiano A., Grazioli V.

Sezione di Microbiologia, "Istituto Clinico Humanitas", Via Manzoni 56, 20090 Rozzano, Milano

Introduzione: nell'etiologia delle vaginiti/vaginosi Gardnerella vaginalis (GV), Candida spp. (CS) e Trichomonas vaginalis (TV) sono i microrganismi chiamati piu' frequentemente in causa; le metodiche di laboratorio classicamente deputate alla loro ricerca (lettura del vetrino dell'essudato vaginale a fresco o dopo colorazione di Gram, coltura in terreni selettivi), oltre a dipendere dalla correttezza di esecuzione del prelievo, presentano, a fronte di un costo contenuto, lo svantaggio di produrre risultati troppo soggetti ad una valutazione soggettiva con tempi che possono arrivare alle 48 ore.

Da pochi anni è disponibile un kit (Affirm VPIII, Becton Dickinson and Company, Sparks,Md.) per la ricerca, mediante ibridazione diretta con sonde DNA specifiche, dei genomi di GV, CS e TV nell'essudato vaginale; la metodica, dotata di adeguata sensibilità analitica, è completata in 40 minuti.

Obiettivo: abbiamo deciso di rivalutare, utilizzando il kit Affirm VPIII, la prevalenza di GV, CS e TV nell'essudato vaginale delle donne che sono giunte alla nostra osservazione sia per sospetta vaginite che per screening routinario prefecondazione assistita.

Materiali e metodi Da gennaio 2002 ad aprile 2003 sono state studiate 2300 donne con età compresa fra i 14 e i 65 anni (650 candidate alla fecondazione assistita e 1655 con disturbi riferibili a vaginite/vaginosi).
Risultati

\begin{tabular}{llll}
\hline ETA' & Gardnerella & Trichomonas & Candida spp \\
\hline$>55(\mathrm{n}=110)$ & $19,1 \%$ & $0,0 \%$ & $7,3 \%$ \\
\hline $55-45(\mathrm{n}=130)$ & $33.8 \%$ & $3,8 \%$ & $16,1 \%$ \\
\hline $35-45(\mathrm{n}=530)$ & $19.6 \%$ & $0,4 \%$ & $9,8 \%$ \\
\hline $25-35(\mathrm{n}=750)$ & $20,4 \%$ & $0,5 \%$ & $12,1 \%$ \\
\hline$<25(135)$ & $25,9 \%$ & $0,7 \%$ & $18,5 \%$ \\
\hline ETA & Gardnerella & Trichomonas & Candida spp \\
\hline $35-45(\mathrm{n}=400)$ & $19,3 \%$ & $0,5 \%$ & $9,0 \%$ \\
\hline $25-35(\mathrm{n}=250)$ & $17,7 \%$ & $1,1 \%$ & $9,3 \%$ \\
\hline
\end{tabular}

\section{Conclusioni}

1) Per quanto riguarda i soggetti sintomatici, pur con i dovuti distinguo relativi all'area geografica di origine, $i$ risultati, almeno per quanto riguarda $\mathrm{GV}$ e $\mathrm{CA}$, sono in linea con quanto riportato dalla letteratura internazionale

\begin{tabular}{llll} 
Autore & \multicolumn{2}{l}{ Gardnerella } & Trichomonas Candida spp \\
\hline Di Bartolomeo S & $23,8 \%$ & $2,4 \%$ & $17,8 \%$ \\
\hline Hong S & $26 \%$ & $4 \%$ & $11 \%$ \\
\hline Acikgoz ZC & $13,8 \%$ & $2,2 \%$ & $26,8 \%$ \\
\hline
\end{tabular}

2) Viene confermato che GV è il più frequente, soprattutto nelle pazienti fra i 45 e i 55 anni

3) Non si evidenziano differenze fra la popolazione sintomatica e quella apparentemente asintomatica

4) Questi primi dati suggeriscono una rivalutazione del ruolo di questi agenti nella genesi delle vaginiti.

\section{G061 \\ TIPIZZAZIONE AUTOMATICA IS6II 0 DI M.TUBERCULOSIS MEDIANTE IL SISTEMA ROBOTIZZATO RIBOPRINTER ${ }^{\circledR}$}

\section{Barreca P.M., Pittaluga F., Marchiaro G., Cirillo D. \\ Laboratorio di Microbiologia Clinica AO San Giovanni Battista, cso Bramante 88, Torino}

L'aumento dei casi di tubercolosi, l'insorgenza e la diffusione di ceppi di M. tuberculosis multiresistenti (MDR), hanno stimolato lo sviluppo di nuove tecniche di tipizzazione molecolare considerate importanti strumenti per la sorveglianza della malattia. L'analisi RFLP delle sequenze di inserzione IS6110 è considerata la tecnica "gold standard" per lo studio epidemiologico della tubercolosi. Nonostante l'elevato potere discriminante, questo metodo presenta lo svantaggio di possedere lunghi tempi di esecuzione e richiede esperienza nell'interpretazione dei patterns. Il RiboPrinter ${ }^{\circledR}$ è un sistema automatico sviluppato per la ribotipizzazione batterica in grado di effettuare anche analisi automatiche in Southern blot. Questo sistema è stato da noi utilizzato per la tipizzazione automatica IS6110 di M. tuberculosis. Lo scopo di questo lavoro è stato quello di valutare la riproducibilità del sistema RiboPrinter ${ }^{\mathbb{B}}$ per la tipizzazione IS6110 automatizzata di M. tuberculosis paragonandolo ai sistemi di tipizzazione manuali.

Sono stati isolati e tipizzati 44 ceppi di M. tuberculosis mediante RFLP-IS6110 convenzionale e PFGE (seguendo i protocolli standard) e mediante RiboPrinter ${ }^{\circledR}$ RFLP-IS6110. Per l'analisi RFLP-IS6110 con RiboPrinter ${ }^{\circledR}$ le cellule sono state trattate con acetone e cloroformio/metanolo (2:1, vol / vol) e risospese meccanicamente in RiboPrinter buffer. L'analisi dei patterns è stata effettuata mediante il software Bionumerics 2.5 per la rivelazione dei clusters.

I risultati ottenuti, analizzati mediante il Software 